\title{
Patrones de variación espacial y temporal de los macroinvertebrados acuáticos en la Laguna de Tecocomulco, Hidalgo (México)
}

\author{
Axel Eduardo Rico-Sánchez ${ }^{1}$, Alexis Joseph Rodríguez-Romero ${ }^{1}$, Eugenia López-López ${ }^{1}$ \& \\ Jacinto Elías Sedeño-Díaz ${ }^{2}$ \\ 1. Intituto Politécnico Nacional, Laboratorio de Bioconservación y Manejo, Escuela Nacional de Ciencias Biológicas, \\ Prol. de Carpio y Plan de Ayala s/n, Col. Santo Tomás, México, D.F., 11340; eulopez@ipn.mx \\ 2. Instituto Politécnico Nacional, Coordinación Politécnica para la Sustentabilidad, Av. Instituto Politécnico Nacional \\ esq. Av. Wilfrido Massieu s/n, Col. Zacatenco, D.F. 07738, México; jsedeno@ipn.mx
}

Recibido 12-XII-2013. Corregido 20-I-2014. Aceptado 13-II-2014.

\begin{abstract}
Spatial and temporal variation patterns in aquatic macroinvertebrates of Tecocomulco Lake, Hidalgo (México). Lake Tecocomulco, Hidalgo, is a relic of the ancient lakes of Anahuac, important for the conservation of resident and migratory birds. However, the composition of aquatic macroinvertebrates is unknown; this is an important gap in conservation as they play an important role in the food web. This study analyzed the spatial and temporal variations in macroinvertebrate assemblages and their relationship with habitat characteristics. We carried out four monitoring campaigns covering the rainy and dry seasons. The monitoring was conducted at six study sites (four in the littoral zone and two in the middle part of the lake), environmental factors were recorded at each study site, water samples were collected for their physical and chemical analysis and aquatic macroinvertebrates were collected. A principal component analysis (PCA) was used to group study sites based on physical and chemical characteristics. Richness of taxa was analysed with rarefaction. We assessed the importance value index of each taxon (considering their frequency of occurrence and abundance). Similarity analyzes were performed between study sites and similarity of taxa with indices of Jaccard and Bray-Curtis, respectively. We performed a canonical correspondence analysis (CCA) between environmental factors and macroinvertebrate taxa. The PCA showed a marked seasonal variation represented by warm periods, with high values of conductivity, alkalinity, hardness, sulfates, and macronutrients ( $\mathrm{N}$ and $\mathrm{P}$ ) and the cold period with low values. We found a total of 26 taxa of aquatic macroinvertebrates and the highest richness was found in August. The Jaccard similarity analysis found differences between the littoral area and the limnetic zone, which differ also in the composition of macrophytes. The littoral zone had the highest taxa richness of macroinvertebrates and macrophytes, while the lowest diversity was found in the offshore zone. The CCA related physicochemical characteristics of the water body with macroinvertebrate taxa showing the influence of both physicochemical characteristics and the composition of macrophytes in the spatio-temporal patterns of aquatic macroinvertebrates in the lake. The dominance of Corixidae highlights a strong grazing activity in the lake and in turn suggests an important amount of food available for higher trophic levels. Our study shows that the macroinvertebrates of Tecocomulco Lake have spatial and seasonal variations that are related to both environmental and biotic factors with groups being dominant. Rev. Biol. Trop. 62 (Suppl. 2): 81-96. Epub 2014 April 01.
\end{abstract}

Key words: wetlands, aquatic macroinvertebrates, relic water body, assemblages, spatio-temporal patterns, Mexico.

Los humedales son sistemas acuáticos que han sido considerados a nivel mundial entre los ecosistemas con mayor diversidad de hábitats (Keeley \& Zedler, 1998; Boeckman \& Bidwell, 2007). México cuenta con 138 humedales inscritos en la ficha de humedales Ramsar que incluyen aquellos de las zonas costeras y continentales a los que se les ha asignado especial importancia como sitios prioritarios para la conservación de aves migratorias (Pérez-Arteaga, Jackson, Carrera \& Gaston, 2005). En estos ecosistemas, las macrófitas juegan un papel 
especial, ya que son de los principales elementos en delinear la estructura física del hábitat, confieren mayor heterogeneidad y modulan los diferentes procesos ecológicos que tienen lugar en los humedales. Además, tienen una fuerte influencia en la abundancia, diversidad y en los atributos de las comunidades de los organismos acuáticos (Fontanarrosa, Chaparro \& O'Farrell, 2012). Asimismo, las condiciones hidrológicas son importantes en la estructura y función de un humedal, ya que modifican los factores ambientales que afectan a la biota que se desarrolla en este ambiente (Mitsch \& Gosselink, 2007).

Los macroinvertebrados acuáticos son un grupo diverso en sistemas de agua dulce, donde desempeñan un papel importante en el reciclamiento de nutrientes y flujo de energía (Gong, Xie \& Wang, 2000). Por ello, son considerados un grupo importante en el monitoreo biológico del ambiente acuático, según Rosenberg \& Resh (1993) y Trigal-Domínguez, FrenándezAláez \& García-Criado (2009) los macroinvertebrados son organismos sedentarios y reaccionan de forma inmediata y predecible ante los impactos humanos en los ecosistemas acuáticos. Adicionalmente, Sharma \& Rawat (2009) señalan que algunos macroinvertebrados responden a cambios específicos en las condiciones de calidad del agua, por lo que han sido empleados como indicadores de la salud de los humedales.

Las comunidades de macroinvertebrados pueden variar en el tiempo y el espacio, pues su diversidad se relaciona con la concentración de nutrientes y productividad del ecosistema, teniendo mayor diversidad los sitios asociados con buena calidad del agua (Latha \& Thanga, 2010). Para entender el papel que juegan los macroinvertebrados en los lagos se requiere conocer la estructura de la comunidad, la dinámica poblacional, las asociaciones con el hábitat, así como los grupos tróficos (Gong, Xie \& Wang, 2000). Particularmente, los estudios en humedales de las zonas tropicales son escasos (Pamplin, Almeida \& Rocha, 2006; Lucca, Pamplin, Gessner, Trivinho-Strixino, SpadanoAlburquerque \& Rocha (2010). La mayoría de los estudios se han enfocado al análisis de macroinvertebrados bentónicos como indicadores de la calidad del agua en ríos (Azrina \& Yap, 2006; Stein, Springer \& Kohlmann, 2008; Lock, Asenova \& Goethals, 2011). Considerando que la diversidad de macroinvertebrados es un indicador de la salud global de un humedal (Sharma \& Rawat, 2009) y que su composición puede presentar fluctuaciones en número de táxones y abundancia por efecto de las condiciones ambientales, en el presente estudio se evaluó la variación espacial y temporal de las comunidades de macroinvertebrados de la Laguna de Tecocomulco, México, humedal inscrito en la Convención Ramsar desde 2003 y su relación con algunas variables ambientales.

\section{MATERIALES Y MÉTODOS}

Área de estudio: La Laguna de Tecocomulco conforma una subcuenca endorreica de origen tectónico volcánico que está ubicada al noreste de la Gran Cuenca del Valle de México, y es considerada el último relicto de los antiguos lagos del Anáhuac (Caballero, Lozano, Ortega, Urrutia, \& Macias, 1999; GarcíaPalomo \& Macías, 2005). Se encuentra a una altitud promedio de $2514 \mathrm{msnm}$. entre las coordenadas $19^{\circ} 53$ '20" $-19^{\circ} 50^{\prime} 08^{\prime}$ 'N y $98^{\circ} 21^{\prime} 54^{\prime}$ '$98^{\circ} 25^{\prime} 44^{\prime \prime} \mathrm{W}$. El clima es templado subhúmedo con lluvias en verano (García, 2004), con una temperatura media anual que va de 12 a $18^{\circ} \mathrm{C}$, la precipitación media anual de un periodo de 30 años es de $700 \mathrm{~mm}$. La profundidad promedio de la laguna es de $2 \mathrm{~m}$, aunque en las zonas más profundas puede alcanzar hasta los $4 \mathrm{~m}$, el área superficial de la laguna alcanza $17.69 \mathrm{~km}^{2}$. De acuerdo con Lot-Helgueras y Novelo-Retana (1978) el espejo de agua está cubierto mayormente por hidrófitas emergentes como Schoenoplectus californicus y Typha latifolia que se encuentran asociadas principalmente a la zona limnética de la laguna y otras del tipo flotante como Potamogeton nodosus, Lemna gibba y otras más que caracterizan a las zonas litorales como Polygonum punctatum y Najas guadalupensis. 
Para el estudio de macroinvertebrados acuáticos de la laguna se eligieron seis sitios de estudio con el propósito de cubrir zonas con características físicas y bióticas (vegetación) contrastantes: el sitio 1 (19 $51^{\prime} 16.74^{\prime \prime} \mathrm{N}-98^{\circ} 23^{\prime} 46.56^{\prime}$ 'W) con presencia de $S$. californicus y $P$. punctatum, y el sitio 2 (19॰51'30.12'N-98²2'58.74” W) asociado sólo a $S$. californicus, se ubican en el interior de la laguna, mientras que los sitios 3 (19 $52^{\prime} 2.82^{\prime \prime} \mathrm{N}-98^{\circ} 24^{\prime} 16.02^{\prime \prime}$

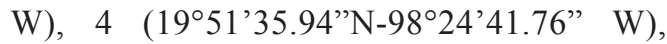

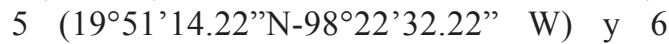
$\left(19^{\circ} 51^{\prime} 53.16^{\prime \prime} \mathrm{N}-98^{\circ} 22^{\prime} 37.68^{\prime \prime} \mathrm{W}\right)$ en la zona litoral. En los sitios 3 a 6, estuvo presente $L$. gibba; en los sitios 4 a 6 estuvo presente $P$. punctatum y en los sitios 3 y 6 estuvieron presentes $P$. foliosus y $S$. californicus. Se realizaron cuatro muestreos abarcando el final de las lluvias en noviembre de 2011 (con influencia de huracanes), la temporada de estiaje en enero y mayo de 2012 (fría y cálida, respectivamente) y el período de lluvias en agosto de 2012.

Variables ambientales: En cada sitio y temporada de estudio se registraron los siguientes factores ambientales in situ: temperatura $\left(\mathrm{T}^{\circ} \mathrm{C}\right)$, oxígeno disuelto (OD $\left.\mathrm{mg} \mathrm{l}^{-1}\right)$, turbidez (NTU), salinidad (UPS) y conductividad $\left(\mathrm{mScm}^{-1}\right)$ con una sonda multiparamétrica Quanta ${ }^{\circledR}$. Estas mediciones fueron realizadas a un metro de profundidad del tirante de agua o menos (zona litoral). Así mismo, en cada sitio se tomaron muestras de agua de $500 \mathrm{~mL}$, por duplicado, que fueron transportadas en obscuridad y refrigeración para su análisis en el laboratorio. Las muestras de agua fueron procesadas mediante las técnicas $\mathrm{HACH} \AA$ para determinar: nitrógeno total $\left(\mathrm{NTmgL}^{-1}\right)$, nitritos $\left(\mathrm{NO}_{2} \mathrm{mgL}^{-1}\right)$, nitratos $\left(\mathrm{NO}_{3} \mathrm{mgL}^{-1}\right)$ nitrógeno amoniacal $\left(\mathrm{NH}_{3} \mathrm{mgL}^{-1}\right)$, sulfatos $\left(\mathrm{SO}_{4} \mathrm{mgL}^{-1}\right)$, ortofosfatos $\left(\mathrm{PO}_{4} \mathrm{mgL}^{-1}\right)$, fósforo total $\left(\mathrm{PTmgL}^{-1}\right)$, color (unidades de $\mathrm{Pt}-\mathrm{Co}$ ) y sólidos suspendidos totales ( $\mathrm{SST} \mathrm{mgL}^{-1}$ ) empleando un espectrofotómetro $\mathrm{HACH}{ }^{\circledR}$ DLR2500. Además se determinó la alcalinidad $\left(\mathrm{CaCO}_{3} \mathrm{mgL}^{-1}\right)$, cloruros $\left(\mathrm{Cl}-\mathrm{mgL}^{-1}\right)$, demanda bioquímica de oxígeno $\left(\mathrm{DBO}_{5} \mathrm{mgL}^{-1}\right)$, dureza total $\left(\mathrm{CaCO}_{3} \mathrm{mgL}^{-1}\right)$, coliformes totales y fecales (NMP100 $\left.\mathrm{mL}^{-1}\right)$ siguiendo las técnicas de Health Association (2005).

Se realizó un análisis de componentes principales (ACP) para ordenar los sitios de estudio con base en sus atributos ambientales (parámetros físicos y químicos), para ello se empleó una matriz de datos con los sitios de estudio y sus parámetros físico-químicos registrados in situ así como otras determinaciones de calidad del agua. Para eliminar el efecto de las diferentes escalas de los factores ambientales analizados se realizó una transformación logarítmica: $\mathrm{X}=\log (x+1)$. A partir del ACP se identificaron las variables ambientales que más contribuyeron en la identificación de patrones de variación espacial y estacional de los sitios de estudio.

Macroinvertebrados acuáticos: En cada sitio y periodo de estudio se recolectaron macroinvertebrados bentónicos con una red tipo D, con abertura de malla de $500 \mu \mathrm{m}$. Se realizaron cinco réplicas por sitio. Los ejemplares colectados se separaron in situ del material abiótico y se fijaron en etanol al $70 \%$ para su preservación y posterior transporte al laboratorio, donde se realizó la separación y determinación taxonómica hasta nivel de familia empleando claves especializadas (Thorp \& Covich, 2001; Merrit, Cummins \& Berg, 2008; Krantz \& Evans, 2009) con ayuda de un microscopio estereoscópico Zeiss modelo Stemi 1000 . La riqueza de táxones fue obtenida contando las familias por sitio y época, se modeló una curva de rarefacción basada en el número de muestras versus número acumulativo de familias de todo el periodo de estudio tal como se ha utilizado por Vance, Smith, Malcolm, Huber \& Bellocq (2007), las curvas de rarefarección se generaron con el programa EstimateS 8.2.0 (Colwell, 2009), así también, se construyó la curva de acumulación de familias por muestra.

Para identificar diferencias estacionales y espaciales en la riqueza de familias los datos fueron examinados usando la prueba de Kolmogorov Smirnov para verificar su ajuste a una 
distribución normal, seguido de un Análisis de Varianza (ANOVA), la prueba de Bartlett para identificar la homogeneidad de las varianzas, y finalmente una prueba post hoc de comparación múltiple de medias Student Newman Keuls con $\alpha=0.05$ y las diferencias se consideraron significativas con $\mathrm{p}<0.05$. Se calculó el Índice de Valor de Importancia (IVI) de cada taxón con la ecuación: $I V I=\% A$ bundancia absoluta $+\%$ de la frecuencia de ocurrencia (Krebs, 1989).

Se realizaron análisis de similitud: uno, de los sitios de estudio considerando la presencia y ausencia de los táxones de macroinvertebrados acuáticos por sitio y periodo de estudio, empleando el índice de Jaccard, y otro de la similitud de las familias considerando su abundancia por sitio y periodo de estudio con el índice de Bray-Curtis. En ambos casos el dendrograma de similitud se construyó por el método de promedios no ponderados (UPGMA).

Se analizó la relación de los macroinvertebrados acuáticos, las macrófitas y los factores ambientales con mayor aportación a la caracterización ambiental de acuerdo con los resultados del ACP, utilizando un análisis de correspondencias canónicas (ACC). Se emplearon dos matrices de datos: 1) de los sitios de estudio y sus atributos físico-químicos y 2) de los sitios de estudio y la composición de táxones de macroinvertebrados y macrófitas. Las matrices de datos fueron transformadas a $\mathrm{X}=\log (x+1)$. Todos los análisis multivariados se realizaron usando XLStat versión 2010. El ACC generó dos gráficos que permiten visualizar A) los sitios de estudio y los vectores de los factores ambientales y B) los sitios de estudio y los atributos biológicos (familias de macroinvertebrados y especies de macrófitas).

\section{RESULTADOS}

Caracterización del hábitat: El ACP de sitios y variables ambientales muestra en sus primeros dos componentes un $43.10 \%$ de varianza explicada (Fig. 1A, B). El diagrama de la ordenación muestra una clara separación estacional de los sitios; los cuadrantes del margen derecho incluyen los periodos cálidos (mayo y agosto, grupos I y II, respectivamente), y los del margen izquierdo el periodo frío (noviembre y enero, grupos III y IV, respectivamente) (Fig. 1A). En el grupo I se ubican los sitios de estudio de mayo, que corresponden al estiaje cálido, caracterizada por los valores más altos de temperatura del agua $\left(18.6-23.6^{\circ} \mathrm{C}\right)$, mayor conductividad $\left(0.401-1.237 \mathrm{mScm}^{-1}\right)$, salinidad (0.18-0.61UPS), sólidos suspendidos totales $\left(31-246 \mathrm{mgL}^{-1}\right)$, alcalinidad (50$\left.435 \mathrm{mgL}^{-1}\right)$, dureza $\left(54-100 \mathrm{mgL}^{-1}\right)$, nitratos (2.1-5.0 $\left.\mathrm{mgL}^{-1}\right)$ у $\mathrm{pH}$ (8.54-9.35) (Fig. 1B), en este mismo grupo se encuentra el sitio 3 del mes de enero. En el grupo II se ubican todos los sitios de estudio en el periodo de lluvias (agosto), este periodo se caracteriza por presentar alta temperatura del aire (14.7$\left.19.6^{\circ} \mathrm{C}\right)$; altos valores de macronutrientes como fósforo total $\left(0.6-1.6 \mathrm{mgL}^{-1}\right)$, ortofosfatos $\left(0.34-2.31 \mathrm{mgL}^{-1}\right)$, nitritos $\left(0.007-0.118 \mathrm{mgL}^{-}\right.$ $\left.{ }^{1}\right)$ y amonio $\left(0.28-2.20 \mathrm{mgL}^{-1}\right)$, y altas concentraciones de sulfatos $\left(1.5-42 \mathrm{mgL}^{-1}\right)$ y de coliformes fecales (220-1600 NMP100 $\left.\mathrm{mL}^{-1}\right)$ y totales (900-1600 NMP100 $\mathrm{mL}^{-1}$ ) (Fig. 1B). El grupo III incluyó principalmente los sitios de muestreo correspondientes al mes de noviembre (final de las lluvias), caracterizado por las más altas concentraciones de nitrógeno total $\left(5.55-12.95 \mathrm{mgL}^{-1}\right)$ y oxígeno disuelto (4.79-7.34mgL-1), pero también las mayores concentraciones de $\mathrm{DBO}_{5}\left(10.20-26.71 \mathrm{mgL}^{-1}\right)$. En el grupo IV se muestran los sitios de estudio durante al estiaje frío (enero), caracterizada por presentar las condiciones opuestas al mes de mayo, es decir, las menores concentraciones de sólidos suspendidos totales $\left(22-178 \mathrm{mgL}^{-}\right.$ $\left.{ }^{1}\right)$, salinidad (0.14-0.17UPS) y conductividad (0.291-0.752 $\left.\mathrm{mgL}^{-1}\right)$, el menor color (10-83 unidades de Pt-Co) y consecuentemente, la menor temperatura del agua $\left(11.35-16.51^{\circ} \mathrm{C}\right)$.

Riqueza de familias: La Laguna de Tecocomulco presentó una riqueza de 26 familias (Cuadro 1) en las 24 muestras (seis sitios y cuatro periodos de estudio). Se considera que este valor es aceptable ya que las curvas de rarefacción y acumulación de familias tienden a hacerse asintóticas (Fig. 2). Las familias están 
A

Sitios (ejes F1 y F2: 43.10\%)

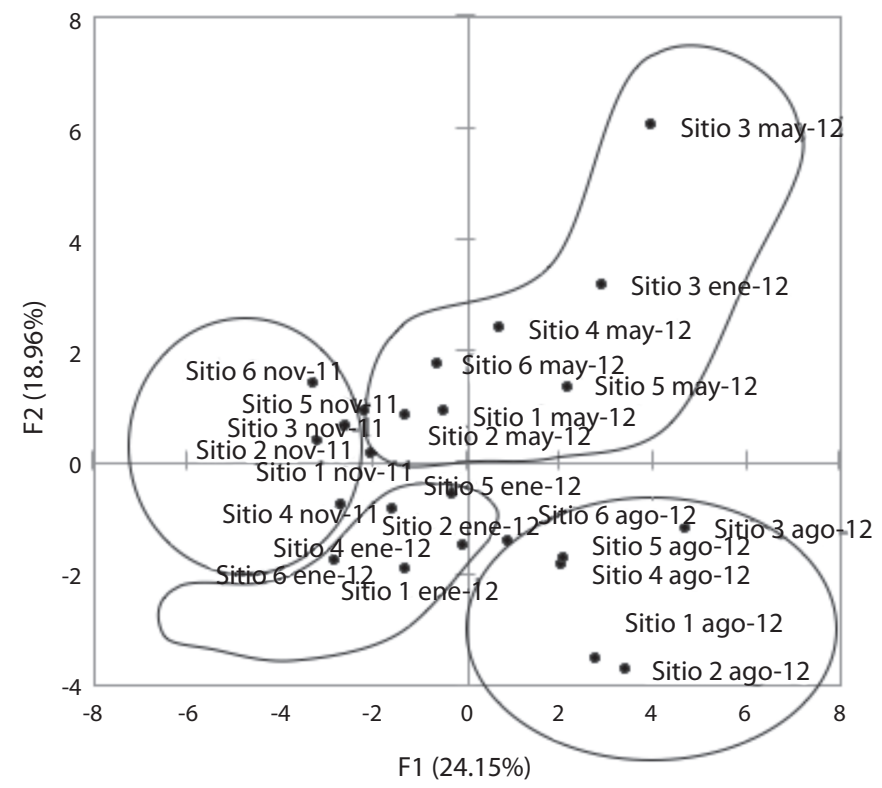

B

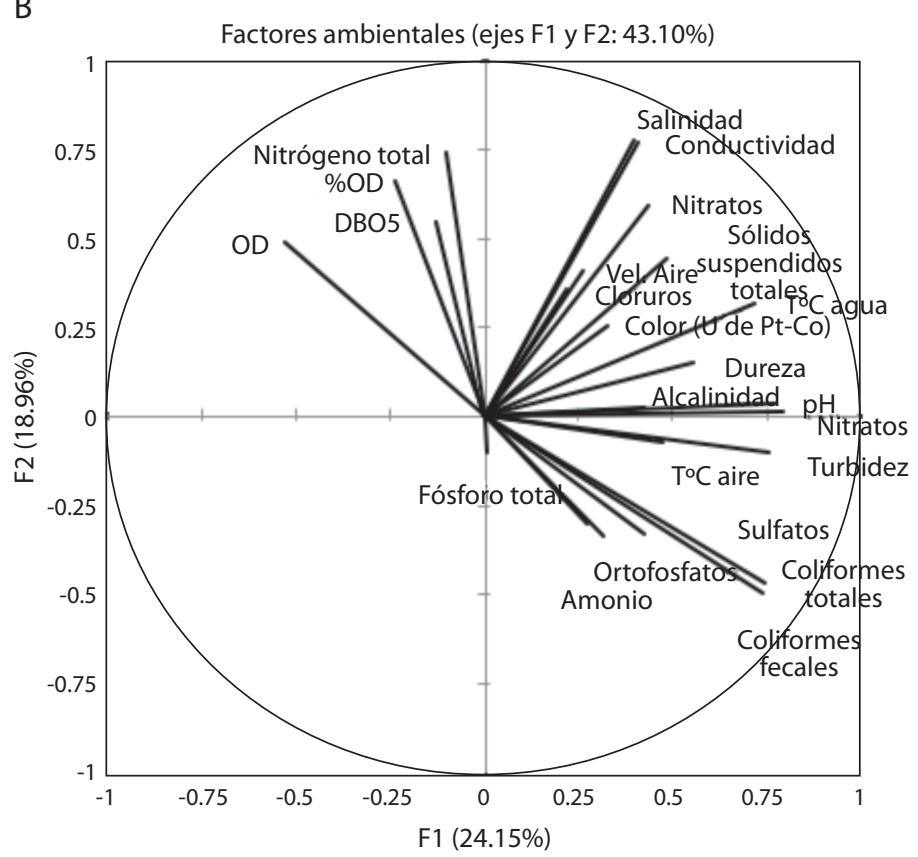

Fig. 1. Diagrama del análisis de componentes principales (ACP) de los sitios de estudio de la Laguna de Tecocomulco. (A) Ordenación de los sitios de estudio y temporada de muestreo, y (B) Vectores de los factores ambientales. Para coadyuvar en la interpretación, los grupos fueron delimitados con líneas curvas.

Fig. 1. Biplot of the principal component analysis (PCA) of study sites at Tecocomulco Lake. (A) Ordination of the study sites and sampling season, and (B) Vectors of environmental factors. The groups were defined with curved lines. 
CUADRO 1

Distribución espacial, temporal e índice de valor de importancia (IVI) de los macroinvertebrados bentónicos de la Laguna de Tecocomulco

TABLE 1

Spatio-temporal distribution and importance value index (IVI) of benthic macroinvertebrates of the Tecocomulco Lake

\begin{tabular}{|c|c|c|c|c|c|c|c|c|c|c|c|c|c|c|}
\hline Phyla & Clase & Orden & Familia & Sitio 1 & Sitio 2 & Sitio 3 & Sitio 4 & Sitio 5 & Sitio 6 & Nov & Ene & May & Ago & IVI \\
\hline AR & IN & $\mathrm{HE}$ & Corixidae & & & & & & & & & & & 57.13 \\
\hline AR & MA & $\mathrm{AM}$ & Hyalellidae & & & & & & & & & & & 26.99 \\
\hline AR & OS & $\mathrm{PO}$ & Notodromadidae & & & & & & & & & & & 16.63 \\
\hline AR & IN & EP & Baetidae & & & & & & & & & & & 13.40 \\
\hline AR & IN & $\mathrm{HE}$ & Notonectidae & & & & & & & & & & & 11.68 \\
\hline AR & IN & DI & Chironomidae & & & & & & & & & & & 11.58 \\
\hline AR & IN & OD & Coenagrionidae & & & & & & & & & & & 11.52 \\
\hline AN & CL & $\mathrm{AH}$ & Hirudinidae & & & & & & & & & & & 8.58 \\
\hline AR & IN & $\mathrm{CO}$ & Hydrophilidae & & & & & & & & & & & 6.69 \\
\hline AR & IN & $\mathrm{CO}$ & Dytiscidae & & & & & & & & & & & 6.19 \\
\hline MO & GA & HY & Planorbidae & & & & & & & & & & & 6.06 \\
\hline MO & GA & BA & Physidae & & & & & & & & & & & 5.80 \\
\hline PL & $\mathrm{TU}$ & TR & Planariidae & & & & & & & & & & & 5.23 \\
\hline AR & $\mathrm{AC}$ & TO & Halacaridae & & & & & & & & & & & 3.46 \\
\hline MO & GA & $\mathrm{HY}$ & Lymnaeidae & & & & & & & & & & & 2.67 \\
\hline AR & IN & OD & Aeshnidae & & & & & & & & & & & 2.20 \\
\hline AR & MA & $\mathrm{DE}$ & Cambaridae & & & & & & & & & & & 1.83 \\
\hline AR & IN & $\mathrm{HE}$ & Belostomatidae & & & & & & & & & & & 1.76 \\
\hline AR & IN & OD & Libellulidae & & & & & & & & & & & 1.63 \\
\hline $\mathrm{AR}$ & IN & $\mathrm{CO}$ & Haliplidae & & & & & & & & & & & 1.17 \\
\hline MO & GA & BA & Ancylidae & & & & & & & & & & & 0.72 \\
\hline AR & IN & $\mathrm{CO}$ & Gyrinidae & & & & & & & & & & & 0.72 \\
\hline AN & $\mathrm{CL}$ & LU & Lumbriculidae & & & & & & & & & & & 0.72 \\
\hline AR & IN & DI & Dixidae & & & & & & & & & & & 0.37 \\
\hline AR & MA & $\mathrm{AM}$ & Gammaridae & & & & & & & & & & & 0.36 \\
\hline \multirow[t]{2}{*}{$\mathrm{AR}$} & IN & DI & Stratiomyidae & & & & & & & & & & & 0.36 \\
\hline & & & Número de taxones & 15 & 12 & 20 & 19 & 22 & 19 & 21 & 19 & 17 & 21 & \\
\hline
\end{tabular}

Phyla: $\mathrm{AR}=$ Arthropoda, $\mathrm{AN}=$ Annelida, $\mathrm{PL}=$ Platyhelminthes, $\mathrm{MO}=$ Mollusca. Clase: $\mathrm{IN}=$ Insecta, $\mathrm{MA}=$ Malacostraca, $\mathrm{OS}=$ Ostracoda, $\mathrm{CL}=$ Clitellata, $\mathrm{GA}=$ Gastropoda, $\mathrm{TU}=$ Turbellaria y $\mathrm{AC}=$ Arachnida. Orden: $\mathrm{HE}=$ Hemiptera, $\mathrm{AM}=\mathrm{Amphipoda}$, $\mathrm{PO}=$ Podocopida, $\mathrm{EP}=$ Ephemeroptera, $\mathrm{DI}=$ Diptera, $\mathrm{OD}=$ Odonata, $\mathrm{AH}=$ Arhynchobdellida $, \mathrm{CO}=\mathrm{Coleoptera}, \mathrm{HY}=$ Hygrophila, $\mathrm{BA}=$ Basommatophora, $\mathrm{TR}=$ Tricladida, $\mathrm{TO}=$ Trombidiformes, $\mathrm{DE}=$ Decapoda y $\mathrm{LU}=$ Lumbriculida.

distribuidas en 14 órdenes, ocho clases y cuatro Phyla. La clase más representada es Insecta con 14 familias. La riqueza de táxones mostró variaciones espaciales y temporales (Cuadro 1). Entre sitios de estudio, los máximos valores de riqueza se presentaron en las zonas litorales, sitios 3 a 6 con 19 a 22 taxones (Student Newman Keuls, $\mathrm{p}<0.05)$, respectivamente; mientras que en el interior de la laguna (sitios 1 y 2 ) alcanzaron 12 y 15 taxones. Por periodo de estudio no se detectaron diferencias significativas (Student Newman Keuls, $\mathrm{p}>0.05$ ), no obstante, los valores máximos ocurrieron en noviembre del 2011 y agosto del 2012 con 21 taxones y el valor mínimo registrado fue en mayo con 17 taxones, seguido de enero con 


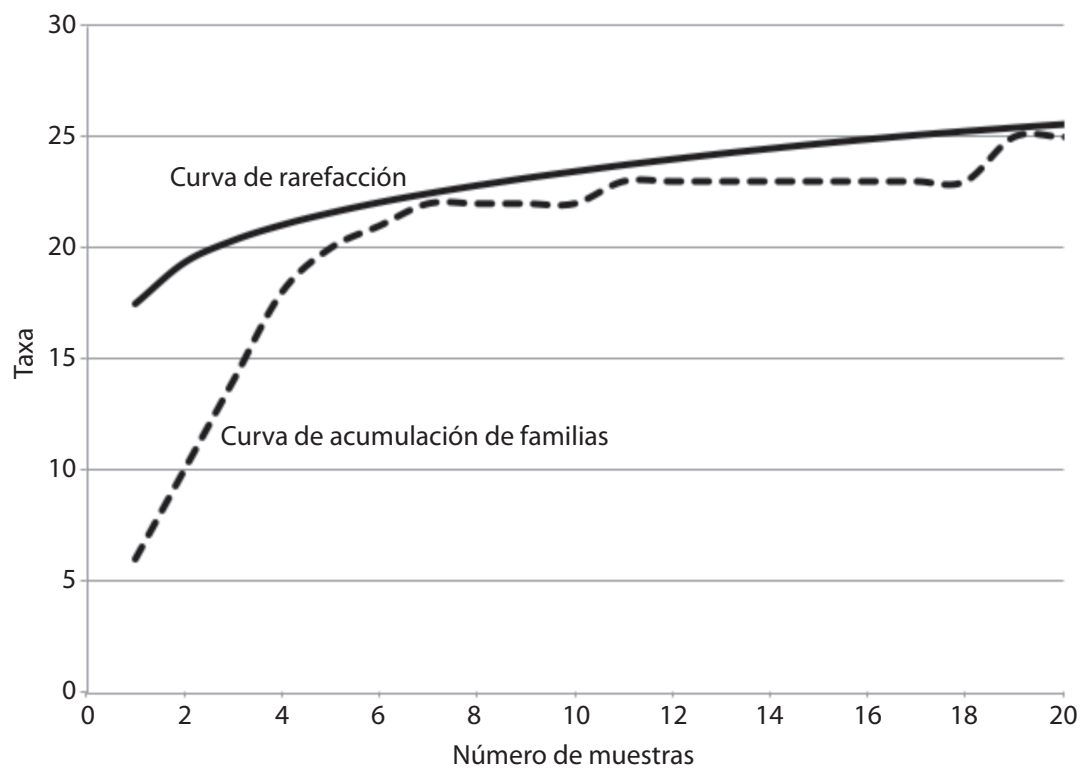

Fig. 2. Riqueza de familias para la Laguna de Tecocomulco durante el período de estudio. Curvas de rarefacción y acumulación de familias.

Fig. 2. Families richness for Tecocomulco Lake during the study period. Rarefaction and accumulation curves of families.

19 táxones. En este sentido, nueve familias estuvieron ausentes durante el estiaje cálido (Belostomatidae, Libellulidae, Haliplidae, Ancylidae, Gyrinidae, Dixidae, Gammaridae, Stratiomyidae, y la clase Oligochaeta) mientras que siete familias no se presentaron en el estiaje frío (Cambaridae, Belostomatidae, Ancylidae, Gyrinidae, Dixidae, Gammaridae y Stratiomyidae) (Cuadro 1).

El IVI representó la frecuencia de ocurrencia y abundancia de los macroinvertebrados de la laguna (Cuadro 1). La familia Corixidae alcanzó la máxima frecuencia y abundancia en todos los sitios de estudio; su valor de IVI fue de 57.13, seguida de Hyalellidae con 26.99, estas dos familias en combinación con la Baetidae, Notonectidae, Chironomidae, Coenagrionidae, Hirudinidae e Hydrophilidae (en total ocho táxones) alcanzaron valores del IVI mayores a 6.69 y se distribuyeron en todos los sitios y periodos de estudio (Cuadro 1). Las familias Physidae, Planaridae y Halacaridae, se encontraron durante todo el periodo de estudio pero solo en los sitios 3 a 6 (zona litoral) con valores del IVI de 3.46 a 5.79. Las familias Lymnaeidae, Aeshnidae y Belostomatidae con valores de IVI de 2.67, 2.2 y 1.76 se distribuyeron tanto en la zona litoral como en la limnética, pero se caracterizaron por su ausencia en noviembre de 2011 (final de las lluvias, inicio de la época fría). Libellulidae y Haliplidae con valores de IVI de 1.63 y 1.17 , respectivamente se caracterizaron por su ausencia durante la época cálida seca. Seis familias ( $23 \%$ del total) alcanzaron valores del IVI menores a $1(0.36$ a 0.72 ), entre ellas hay grupos que sólo se encontraron en el interior de la laguna (sitios 1 y 2) y sólo se encontraron en el periodo de frío (Ancylidae y Gammaridae) o algunos que se distribuyeron tanto en el litoral como en el interior de la laguna pero sólo estuvieron presentes en las lluvias (Gyrinidae y Dixidae).

Similitud entre sitios y épocas de muestreo: El análisis de similitud entre sitios y épocas de estudio por la presencia-ausencia de los táxones permitió la identificación de dos grupos a un nivel de truncamiento de 0.43 (I y II), que 


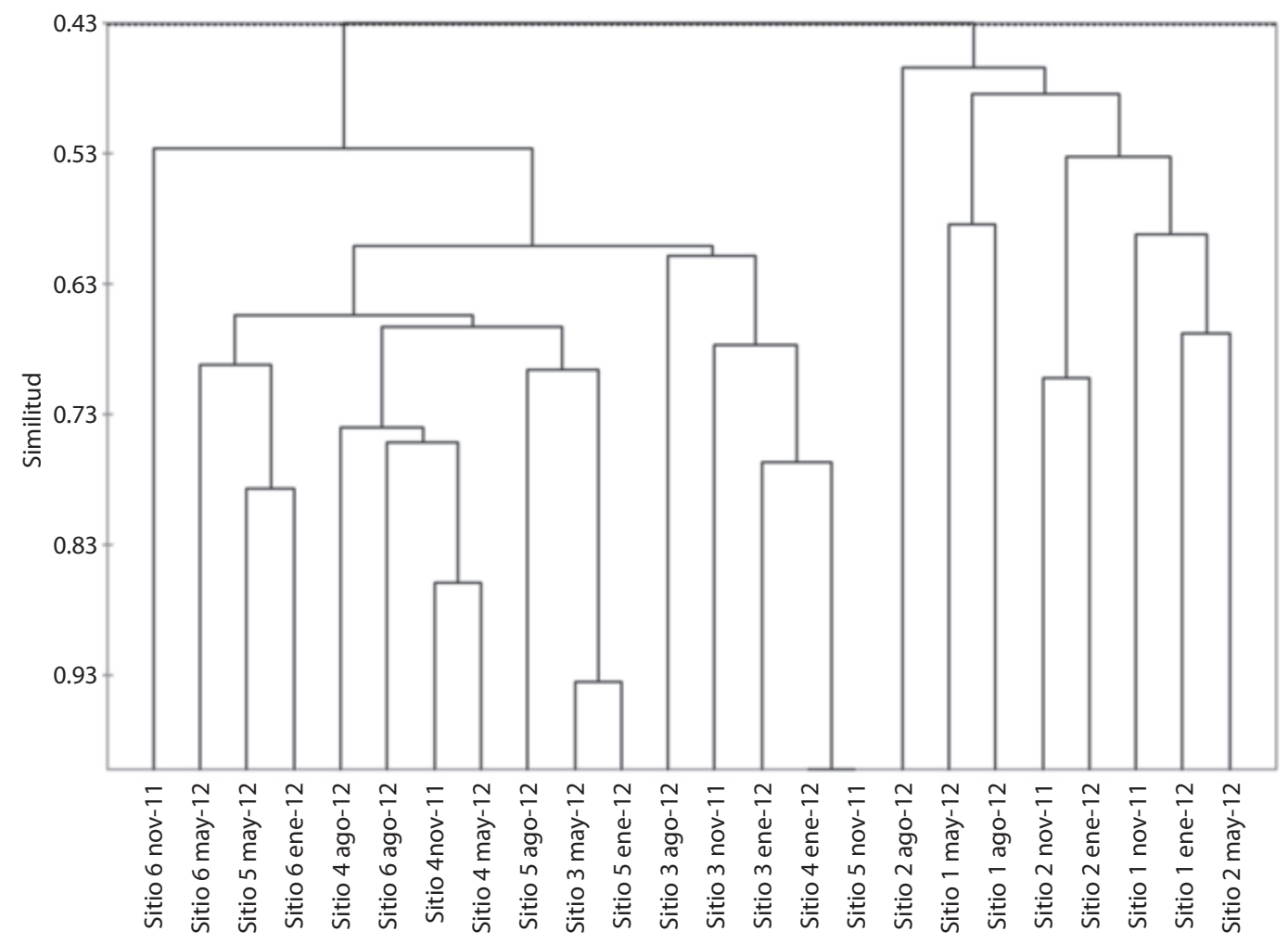

Fig 3. Dendrograma de similitud (UPGMA) con el índice de Jaccard de los sitios de estudio en la Laguna de Tecocomulco, basado en la presencia y ausencia de las familias de macroinvertebrados bentónicos (con un nivel de corte igual a 0.43 ). Fig. 3. Cluster of similarity (UPGMA) with the Jaccard index of the study sites in the Tecocomulco Lake, based on the presence and absence of benthic macroinvertebrate families (with a cutoff at 0.43 ).

diferencian espacialmente a la laguna (Fig. 3). El grupo I incluye a los sitios 3, 4, 5 y 6 , la zona litoral, mientras que el grupo II incluye a los sitios 1 y 2 ubicados en la zona limnética de la laguna. Al interior de cada grupo se pueden identificar subgrupos de sitios de estudio correspondientes al periodo frío (noviembre y enero) y al cálido (mayo y agosto).

Similitud en composición de familias: El análisis de similitud de Bray-Curtis mostró las asociaciones entre los diferentes taxones y evidenció cinco grupos (I, II, III, IV, y V) a un nivel de truncamiento de 1.25 (Fig. 4) que denotan diferencias espaciales y temporales en la distribución de los macroinvertebrados de Tecocomulco. El grupo I lo integran Planariidae,
Dytiscidae, Coenagrionidae, Notonectidae, Baetidae, Planorbidae, Chironomidae, Physidae e Hirudinidae que se presentaron en todos los sitios y periodos de estudio y alcanzaron valores altos del IVI (13.40-5.23). El grupo II incluye a Haliplidae, Libellulidae, Lymnaeidae, Halacaridae e Hydrophilidae que tienen alta frecuencia de ocurrencia pero cuya abundancia no fue alta, se distribuyen predominantemente de la zona litoral y están presentes en las épocas cálidas seca y húmeda. El grupo III está representado por Ancylidae y Gammaridae que se localizan sólo en los sitios del interior de la laguna y en la época fría. El grupo IV reunió a Notodromadidae, Hyalellidae y Corixidae, estos táxones se caracterizaron por presentar la mayor distribución espacial y temporal, 


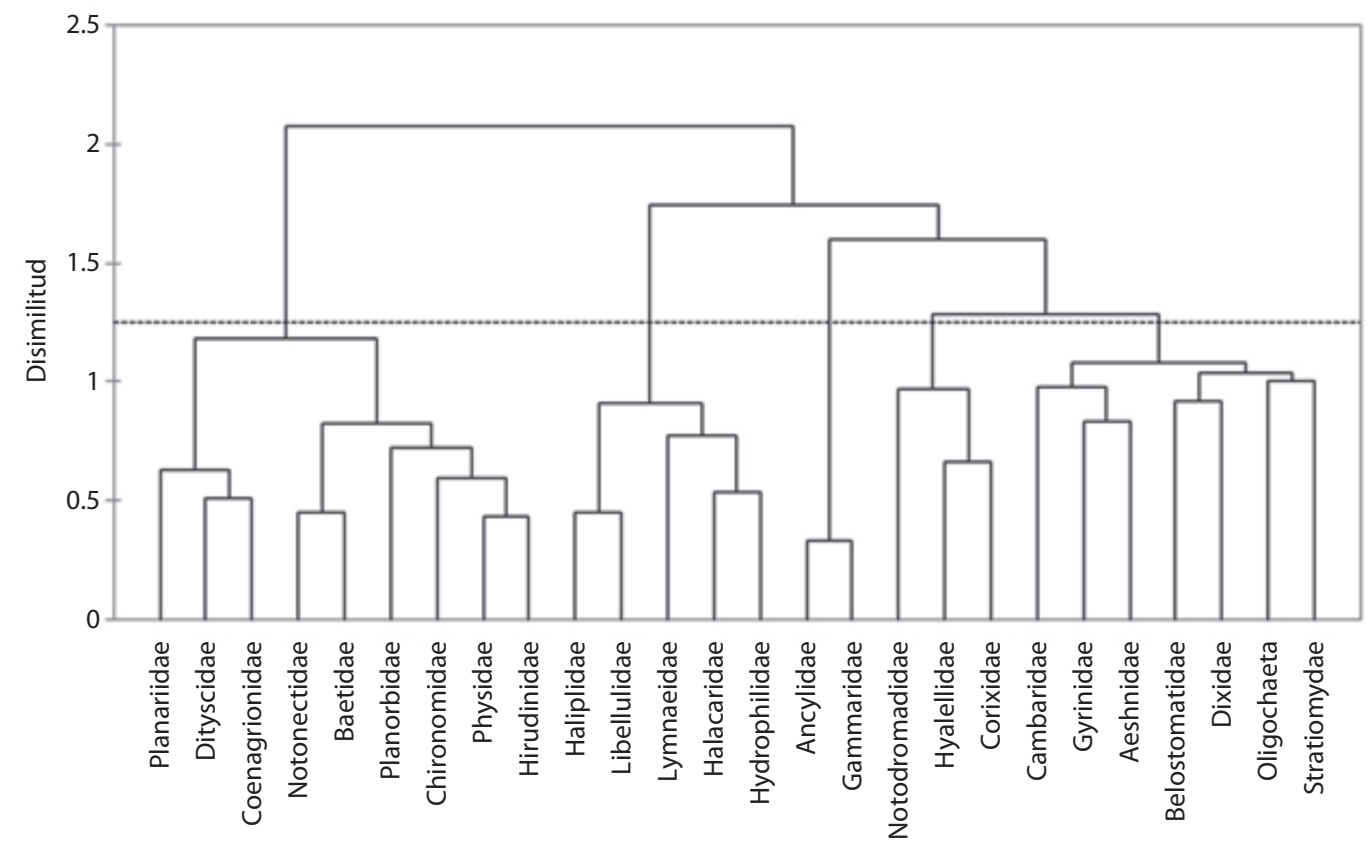

Fig. 4. Dendrograma de similitud (UPGMA) con el índice de Bray-Curtis que muestra la relación de los diferentes grupos taxonómicos que se encontraron en la laguna. Se observan cinco grupos por su relación de ocurrencia y abundancia en los sitios de estudio.

Fig. 4. Cluster of similarity (UPGMA) with the Bray-Curtis index showing the relationship of the different taxonomic groups found in the lake. There are five groups for their occurrence and abundance relationship in the study sites.

además de que su abundancia fue la mayor por lo que alcanzaron los valores más altos del IVI (57.13-26.99) (Cuadro 1), es decir, las familias de macroinvertebrados dominantes. El último grupo, el V se conformó por Cambaridae, Gyrinidae, Aeshnidae, Belostomatidae, Dixidae, Lumbriculidae y Stratiomyidae, caracterizados por su escasa abundancia y ocurrencia ya que fueron taxones poco representados en la laguna y con una distribución restrictiva.

Relación entre familias y características del hábitat: Para identificar las relaciones entre las características del hábitat, y las familias de macroinvertebrados se aplicó un ACC que alcanzó un $40.31 \%$ de varianza explicada en sus dos primeros componentes. En este análisis (Fig. 5A, B) se observa una clara separación espacial y estacional. Los sitios 1 y 2 , del interior de la laguna quedaron ubicados en el extremo derecho del diagrama (Fig.
$5 \mathrm{~A}$ ), caracterizados por altos valores de $\mathrm{DBO}_{5}$ y sulfatos y las macrófitas Schoenoplectus californicus y Polygonum punctatum y con el dominio de Corixidae y Hyalellidae. Mientras que la zona litoral, sitios 3 a 6 , se ubicaron en un gradiente con efecto estacional: las épocas cálidas seca y lluviosa, se localizan, en los cuadrantes inferiores relacionadas con valores altos de parámetros fisicoquímicos como coliformes fecales, $\mathrm{DBO}_{5}, \mathrm{pH}$, dureza y temperatura del agua (Fig. 5A) y con una comunidad integrada por Notonectidae, Belostomatidae, Gyrinidae, Dixidae, Hirudinidae, Notodromadidae, Cambaridae, Coenagrionidae y Corixidae. Estos taxones están asociados también a las macrófitas Lemna gibba y Polygonum punctatum, que caracterizan principalmente a los sitios litorales (Fig. 5B). El final de las lluvias (noviembre 2011) y el estiaje frío (enero 2012) se localizan en los cuadrantes superiores con relación a factores ambientales 


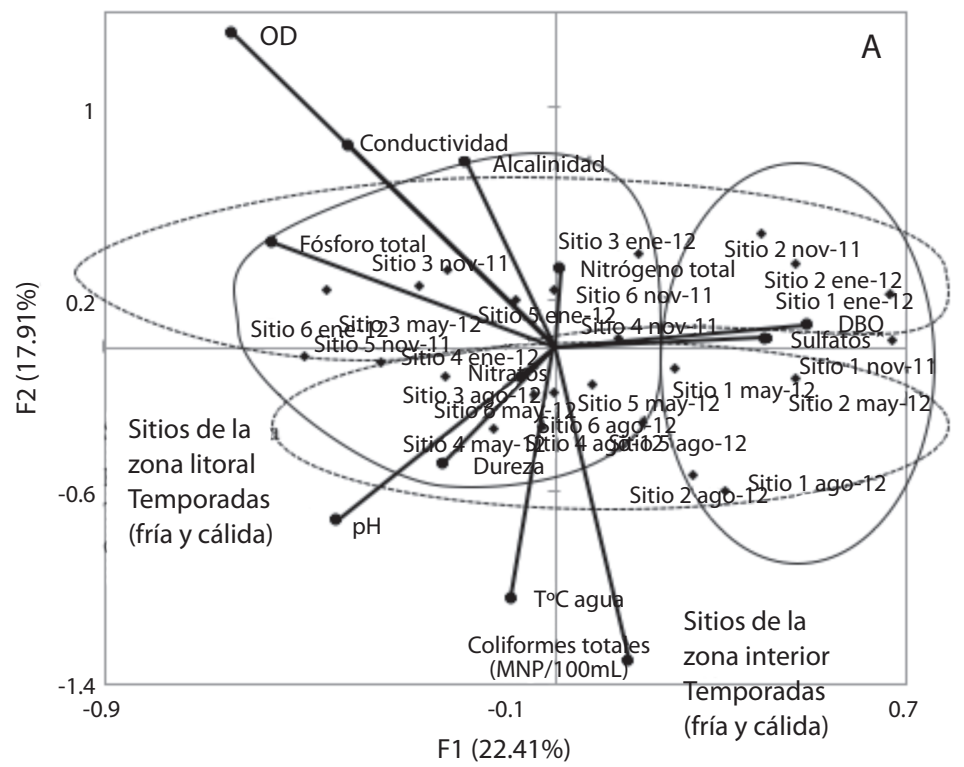

Ejes F1 y F2: $40.31 \%$

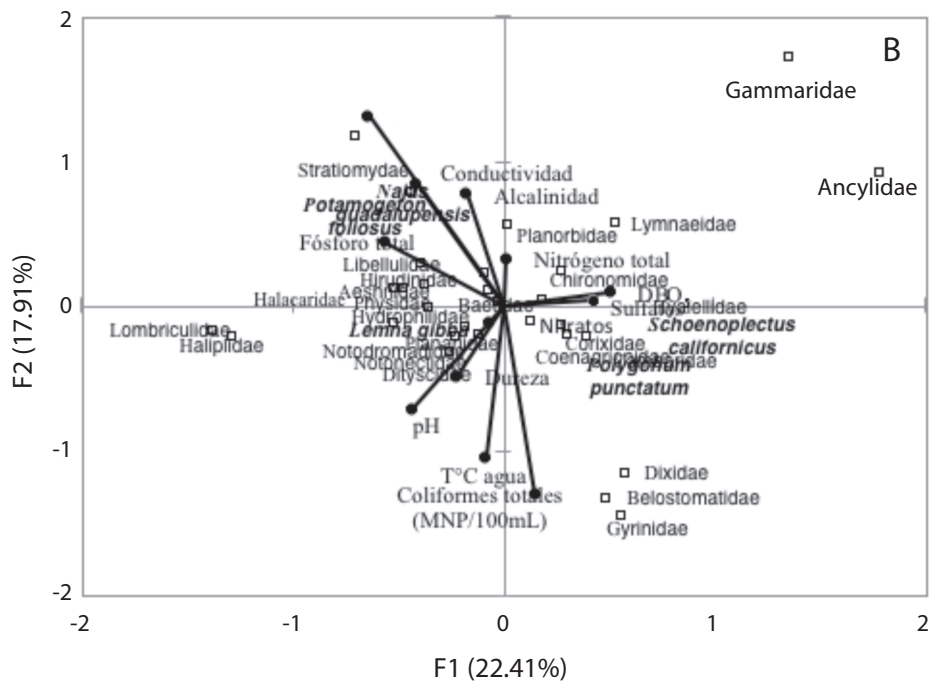

Fig. 5. Diagrama del análisis de correspondencias canónicas (ACC), A) Relación de las variables ambientales respecto a los sitios y temporadas de muestreo y B) Relación de los factores ambientales con los atributos biológicos (familias de macroinvertebrados y especies de macrófitas).

Fig. 5. Biplot of canonical correlation correspondence analysis (CCA), A) Relationship between environmental variables with study sites and sampling periods and B) Relationship between environmental factors with biological attributes (families of macroinvertebrates and macrophyte species). 
con alto valor de OD $\left(4.63-8.52 \mathrm{mgL}^{-1}\right)$, fósforo total $\left(0.44-3.30 \mathrm{mgL}^{-1}\right)$, conductividad $\left(0.258-0.752 \mathrm{mScm}^{-1}\right)$ y nitrógeno total (5.55$\left.12.95 \mathrm{mgL}^{-1}\right)$ relacionado con la presencia de las familias Planorbidae, Halacaridae, Gammaridae, Aeshnidae, Physidae, Chironomidae, Stratiomyidae, Lymnaeidae, Libellulidae, Planariidae, Ancylidae, Haliplidae, Hyalellidae y Oligochaeta, estos táxones están asociados a la presencia de Najas guadalupensis y Potamogeton foliosus, principalmente.

\section{DISCUSIÓN}

La Laguna de Tecocomulco presenta una clara estacionalidad, esto se evidencia en la segregación de los sitios de estudio por época de muestreo observado en el ACP. Los grupos I y II representan las épocas cálidas (seca y húmeda, respectivamente) caracterizadas por altas concentraciones de sulfatos, ortofosfatos, salinidad y conductividad (Fig. 1B). En este grupo se ubicó el sitio 3, que por tratarse de una zona litoral se presentó como una charca aislada para el mes de enero, lo que explica los valores altos de conductividad $\left(0.752 \mathrm{mScm}^{-1}\right)$ y salinidad (0.36UPS) y le confiere características similares a las del periodo de estiaje. Existe evidencia de los cambios climáticos que ha sufrido la Laguna de Tecocomulco y se ha detectado la presencia de estratos calcáreos en sus sedimentos que dan cuenta de épocas donde el ambiente fue sumamente árido (Caballero, Lozano, Ortega, Urrutia, \& Macias 1999). Esto a su vez puede tener influencia sobre sus elevadas concentraciones de salinidad y conductividad. Sin embargo, dada la naturaleza de la Laguna de Tecocomulco (endorreica y somera) la conductividad y la concentración de iones se incrementa durante la época cálida seca principalmente por efecto de la evapotranspiración. En los humedales la pérdida de agua hacia la atmósfera ocurre desde el suelo, el agua misma del humedal, y las porciones aéreas de las macrófitas emergentes (Kandlec \& Knight, 1996). Jeglum, Sandring, Christensen, Glimskär, Allard, Nilsson \& Svensson (2011) señalan que las temperaturas durante el verano promueven la evapotranspiración disminuyendo la profundidad de los humedales; estos autores encontraron un incremento de la conductividad durante las estaciones cálida y lluviosa. En la Laguna de Tecocomulco, la conductividad y otros iones también se incrementan durante la época cálida (mayor evapotranspiración) y la lluviosa (acarreo de materiales alóctonos); esto último tiene como consecuencia que al final de las lluvias se presenten los valores más altos de $\mathrm{DBO}_{5}$ como resultado de todo el material que llega de la cuenca de captación. En el estudio de Jeglum, Sandring, Christensen, Glimskär, Allard, Nilsson \& Svensson (2011) al igual que en el presente, durante la época fría se presentaron las más bajas concentraciones de conductividad, alcalinidad, y temperatura del agua. Lo anterior pone de manifiesto que las variaciones estacionales son importantes en la Laguna de Tecocomulco. De la Lanza \& HernándezPulido (2005) señalaron que en Tecocomulco la tendencia del $\mathrm{pH}$ hacia lo alcalino está asociada al aporte de las sales disueltas de la subcuenca después de los escurrimientos de la época de lluvias, o también a la alta producción primaria de la vegetación que consume $\mathrm{CO}_{2}$ durante el día, lo que incrementa el $\mathrm{pH}$.

Los estudios sobre macroinvertebrados acuáticos en sistemas lénticos de México dentro de la zona tropical son escasos. Pamplin, Almeida, \& Rocha (2006) en un estudio realizado en Brasil, en el embalse Americana $\left(13.8 \mathrm{Km}^{2}\right.$ de área superficial) en 15 sitios de estudio y dos períodos (estiaje y lluvias), señalan una riqueza de ocho táxones (a nivel de familia y/o orden) con representación de 19 géneros. Por otro lado, Lucca, Pamplin, Gessner, Trivinho-Strixino, Spadano-Alburquerque \& Rocha (2010) en un estudio de sólo dos épocas (lluvias y estiaje) y 20 sitios, encontraron también una riqueza de ocho táxones (a nivel de familia y/o orden) representando 23 y 31 géneros para cada época, respectivamente, para el lago Caçó (aproximadamente $2.5 \mathrm{Km}^{2}$ ). Mientras que para la laguna de Tecocomulco (con una extensión de $17.69 \mathrm{Km}^{2}$ ) en sólo seis sitios de estudio y cuatro épocas de monitoreo 
se encontraron 26 táxones. Las curvas de rarefacción y acumulación de táxones (Fig. 2), dada su tendencia asintótica, indican que el número de táxones para la Laguna de Tecocomulco está muy cercano al máximo que se podría encontrar. Al igual que los estudios de Pamplin, Almeida, \& Rocha (2006) y Lucca, Pamplin, Gessner, Trivinho-Strixino, SpadanoAlburquerque \& Rocha (2010), la clase más representada dentro de las familias encontradas en la Laguna de Tecocomulco es Insecta. Sin embargo, en este estudio se presentó una mayor diversidad de familias representantes de la Clase Insecta (14 familias), mientras que en el estudio de Pamplin, Almeida, \& Rocha (2006), se encontraron sólo dos familias (Diptera y Ephemeroptera), y en el estudio de Lucca, Pamplin, Gessner, Trivinho-Strixino, SpadanoAlburquerque \& Rocha (2010) sólo se encontraron representantes de la familia Diptera. Lo anterior indica que la Laguna de Tecocomulco presenta un mayor número de taxones (a nivel de familia) que otros cuerpos de agua de la zona intertropical.

Los componentes mayoritarios de la comunidad de macroinvertebrados acuáticos, de acuerdo con los resultados del IVI fueron de la clase Insecta, la familia Corixidae fue la más dominante (IVI de 57.13), seguido de la familia Hyalellidae (Crustacea) con un IVI de 26.99. En conjunto estas dos familias con Baetidae, Notonectidae, Chironomidae, Coenagrionidae, Hirudinidae e Hydrophilidae presentaron valores de IVI $>6$, y estuvieron presentes prácticamente en todas las épocas y sitios de estudio. Estas familias siempre estuvieron asociados con la presencia de vegetación acuática, tal como lo mencionan Savage (1990) y Van den Berg, Coops, Meijer, Scheffer \& Simons (1998). El sitio de estudio con la menor diversidad de vegetación fue el sitio 2 , en donde sólo se observó la presencia de Schoenoplectus californicus. Las familias Notodromadidae y Dytiscidae a pesar de presentar valores de IVI $>6$, estuvieron ausentes en el sitio 2, aunque presentes en todas las épocas de estudio en los demás sitios. En particular los coríxidos son considerados consumidores primarios en los ambientes acuáticos. Estos organismos al ser dominantes en la laguna de Tecocomulco ponen de manifiesto el estado trófico del cuerpo de agua y revelan una alta productividad primaria que a su vez, genera una alta disponibilidad de alimento para niveles tróficos superiores, constituyendo una fuente de alimento para otros organismos acuáticos como el caso de algunas especies de aves acuáticas. Barahona, Millan \& Velasco (2005) señalan que los coríxidos son importantes consumidores y a su vez son depredados por otros invertebrados y vertebrados acuáticos (Dytiscidae, peces y aves acuáticas).

Los resultados del estudio permitieron identificar dos zonas, en particular el análisis de similitud de Jaccard permitió separar dos regiones de la laguna, la zona litoral (grupo I), de la del interior de la laguna (grupo II). Cada una de estas zonas cuenta con características particulares. La zona litoral es la que tiene la mayor interacción con el ambiente terrestre y recibe los impactos directos de las actividades agrícola y pecuaria que se realizan en las zonas adyacentes a la laguna; esta zona se caracteriza por una gran diversidad de macrófitas, tal como lo indican Lot \& Novelo (1978) y Caballero, Lozano, Ortega, Urrutia, \& Macias (1999). Thomaz \& Cunha (2010) señalan que el incremento en la abundancia, diversidad y la composición de comunidades de diferentes grupos de organismos adheridos a la vegetación está fuertemente influenciada por la complejidad de macrófitas en diferentes escalas espaciales. Los mismos autores plantean que el incremento en abundancia y diversidad de organismos acuáticos en el litoral se puede atribuir a varias razones entre las que destacan: que las macrófitas proveen de sustrato para el establecimiento de un mayor número de táxones, además de que constituyen un refugio y también proveen de diferentes tipos de alimento (perifiton). Estas características pueden estar operando en las macrófitas litorales de la Laguna de Tecocomulco. Por su parte en el interior de la laguna se encuentran predominantemente especies de macrófitas emergentes $S$. californicus y Typha latifolia (Caballero, Lozano, Ortega, 
Urrutia, \& Macias 1999), especies que por sus características le confieren menor complejidad al hábitat, por lo que los resultados de la agrupación de los sitios de estudio con base en la presencia y ausencia de macroinvertebrados sugieren que las especies de macrófitas del litoral son capaces de soportar comunidads de macroinvertebrados más diversas que las de la zona limnética.

Los macroinvertebrados de Tecocomulco también presentan diferencias espaciales y temporales. Los grupos I y IV (organismos dominantes), usando el índice Bray Curtis, están conformados por táxones que se distribuyen en toda la laguna y durante todo el período de estudio, lo cual puede estar asociado con la heterogeneidad de hábitat que presenta la laguna y que garantiza la permanencia de éstos. Dicha heterogeneidad está dada por las macrófitas, que de acuerdo con Thomaz \& Cunha (2010), éstas proporcionan alimento, refugio, áreas para colonización y reproducción, y promueven un incremento en la biodiversidad y las relaciones inter e intra específicas. De igual forma, la presencia de grupos con bajas densidades y/o frecuencias de ocurrencia (espacial o temporal), pone de manifiesto las estrechas tolerancias de los organismos a condiciones que pudieran no ser favorables para ellos (Grupos II, III y V). Así también, Closs, Downes $\&$ Boulton (2004) señalan que los efectos del ambiente influyen directamente sobre los hábitats donde viven los macroinvertebrados, y que la tolerancia a éstos es diferente en cada grupo. Así las condiciones físicas y químicas varían tanto espacial como temporalmente de tal forma que ciertos táxones pueden ser excluidos de hábitats con bajas concentraciones de oxígeno disuelto, mientras que otros pueden sobrevivir aún en condiciones de hipoxia debido a las características adaptativas que éstos presentan tales como sus modos respiratorios con numerosas branquias (Ephemeroptera), o abundante hemoglobina (Chironomidae) e incluso respiración con oxígeno atmosférico (Coleoptera y Díptera, organismos que cuentan con sifón).

En general, los resultados ponen de manifiesto que es posible identificar una segregación temporal basada principalmente en la temperatura del agua (que fue evidenciado por el ACP y el ACC). Mientras que la variación espacial está en función de la presencia y tipo de macrófitas (que se mostró en el análisis de similitud de Jaccard por sitios). Lo anterior confirma que los macroinvertebrados presentan patrones de variación espaciales y temporales influenciados por los factores abióticos y bióticos. La zona litoral se caracterizó por una mayor riqueza de macrófitas y macroinvertebrados, Agostinho, Gomes \& Julio (2003) y Fontanarrosa, Chaparro \& O'Farrell (2012) mencionan que la presencia de macrófitas acuáticas reduce la depredación, proporciona alimento, refugio y genera ambientes oxigenados aumentando el número de espacios disponibles para los macroinvertebrados, en especial de los órdenes Coleoptera y Hemiptera promoviendo una mayor diversidad de macroinvertebrados. La zona limnética se caracterizó por presentar una menor cantidad de diversidad vegetal (Lot-Helgueras \& Novelo-Retana, 1978) y no presenta las condiciones más aptas para el desarrollo de macroinvertebrados ya que de acuerdo con De la Lanza \& Hernández-Pulido (2005) esta zona se caracteriza por tener mayor nivel de profundidad (profundidad máxima de $4 \mathrm{~m}$ ) y una zona anóxica (los valores mínimos de oxígeno disuelto detectados fueron de $2.22 \mathrm{mgL}^{-1}$ y máximos de $4.2 \mathrm{mgL}^{-1}$ ), que evita la proliferación de otros grupos a diferencia de Chironomidae y Corixidae como ya se ha discutido. El patrón temporal se caracterizó por proporcionar condiciones a los macroinvertebrados para su ocurrencia en diferentes temporadas, pues en el caso de la temporada de lluvias que mostró altos niveles de coliformes fecales, $\mathrm{DBO}_{5}, \mathrm{pH}$ y temperatura, es debida a que la zona tiene un gran impacto agrícola y ganadero. Por lo que el efecto de la lluvia ejerce un lavado en los campos externos a la laguna y gracias a la naturaleza endorreica de la subcuenca de Tecocomulco todos los contaminantes van directo hacia ella y con esto el aumento de las concentraciones de coliformes fecales y la proliferación de otros agentes bacterianos, así como la entrada de otros iones con origen en 
el uso de pesticidas (Aguilar-Martínez, PrietoGarcía, Román-Gutiérrez, Monks-Sheets \& Pulido-Flores, 2007) y nutrientes orgánicos e inorgánicos que provocan un $\mathrm{pH}$ alcalino. $\mathrm{Al}$ final de la temporada de lluvias y estiaje frío se encontraron altos niveles de OD, fósforo total, conductividad y nitrógeno total que permitieron la máxima ocurrencia de un grupo de taxones. El oxígeno disuelto es mayor a bajas temperaturas (Buentello, Gatlin \& Neill, 2000), por lo que el descenso de temperatura de la temporada fría provocó altas concentraciones de OD.

Los macroinvertebrados acuáticos de la Laguna de Tecocomulco presentaron variaciones estacionales que responden principalmente a las épocas cálida (seca y húmeda) y fría, con una mayor diversidad durante la época cálida y lluviosa (verano). Así mismo, existen diferencias significativas entre la comunidad de la zona litoral con respecto a la que se desarrolla en la zona interior de la misma, con una clara influencia de las macrófitas presentes. La laguna está dominada por tres familias: Corixidae, Hyalellidae y Notodromadidae, que en conjunto suman el $50 \%$ del valor total que alcanza el IVI. Existe un componente de ocho táxones que incluye a las familias dominantes, que son residentes permanentes de toda la laguna, mientras que se presentan grupos exclusivos de ciertas zonas y épocas del año. Los coríxidos al ser dominantes sugieren una fuerte herbivoría en la laguna y a su vez constituyen una fuente de alimento disponible para niveles tróficos superiores. Los resultados indican que la Laguna de Tecocomulco tiene variaciones espaciales $\mathrm{y}$ temporales relacionadas tanto a factores ambientales como bióticos con la presencia de grupos dominantes. En ese sentido y considerando su diversidad de macroinvertebrados, la Laguna de Tecocomulco debe ser sujeta a un plan de conservación y manejo.

\section{AGRADECIMIENTOS}

Al Instituto Politécnico Nacional por el financiamiento de los proyectos: a) Evaluación de la salud del humedal "Laguna de
Tecocomulco", Hidalgo (sitio RAMSAR), clave 20111126; b) Indicadores biológicos en sistemas lénticos, un acercamiento multimétrico en Tecocomulco, Hidalgo: Caso de estudio, clave 20120931; y c) Los macroinvertebrados de la Laguna de Tecocomulco, Sitio RAMSAR, como indicadores de la calidad del agua, clave 20110602.

\section{RESUMEN}

La Laguna de Tecocomulco (Hidalgo) es un relicto de los antiguos Lagos del Anáhuac con suma importancia para la conservación de aves acuáticas. No obstante, se desconoce su composición de macroinvertebrados. En el presente estudio se analizaron las variaciones espaciales y temporales de los macroinvertebrados acuáticos de la laguna. Se realizaron cuatro campañas de monitoreo (lluvias y estiaje). Se estudiaron seis sitios (litorales y en interior de la laguna), se registraron factores ambientales, se determinaron parámetros de calidad del agua y se recolectaron macroinvertebrados acuáticos. Se obtuvo la riqueza de familias y se calculó su Índice de Valor de Importancia. Se realizaron análisis multivariados de ordenación por componentes principales (ACP) con base en sus características físicas y químicas y de similitud entre sitios y familias con los índices de Jaccard y Bray-Curtis. Tambien se hizo un análisis de correspondencias canónicas (ACC) de factores ambientales y macroinvertebrados acuáticos y macrófitas. El ACP mostró la variación estacional, con el período cálido (mayo y agosto) y el periodo frío (noviembre y enero) mostrando altos valores de conductividad, alcalinidad, dureza, sulfatos y macronutrientes (N y $\mathrm{P}$ ). Se encontraron 26 familias de macroinvertebrados, con la máxima riqueza en agosto. El análisis de similitud de Jaccard diferenció los sitios litorales por su mayor riqueza de familias de la zona limnética, mismos que presentan diferencias en la composición de macrófitas. El estudio revela que la Laguna de Tecocomulco tiene variaciones espaciales y temporales relacionadas tanto con factores ambientales como bióticos con la presencia de grupos dominantes. En ese sentido, y considerando su diversidad de macroinvertebrados, la Laguna de Tecocomulco debe ser sujeta a un plan de conservación y manejo.

Palabras clave: humedales, macroinvertebrados acuáticos, "ensambles", comunidades, cuerpo de agua relicto, patrones espacio temporales, México.

\section{REFERENCIAS}

Agostinho, A. A., Gomes, L. C., \& Julio, H. F. (2003). Relaçies entre macrófitas aquáticas e fauna de peixes. In S. M. Thomaz \& L. M. Bini. (Eds.), Ecologia 
e Manejo de Macrófitas Aquáticas (pp. 261-280). Paraná, PAR: EDUEM.

Aguilar-Martínez, J., Prieto-García, F., Román-Gutiérrez, A. D., Monks-Sheets, W. S. \& Pulido-Flores, G. (2007). Niveles de pesticidas organoclorados en Cyprinus carpio (Linnaeus, 1758) del lago de Tecocomulco. Hidalgo, México: Universidad Autónoma del Estado de Hidalgo.

Health Association. (2005). Standard methods for the examination of wáter and wastewater. Washington, DC: APHA, AWWA \& WEF

Azrina, M. Z. \& Yap, C. K. (2006). Anthropogenic impacts on the distribution and biodiversity of benthic macroinvertebrates and water quality of the Langat river, Peninsular Malaysia. Journal of Ecotoxicology and Environmental Safety, 64(3), 337-347.

Barahona, J., Millan, A., \& Velasco, J. (2005). Population dynamics, growth and production of Sigara selecta (Fieber, 1848) (Hemiptera, Corixidae) in Mediterranean hypersaline stream. Freshwater Biology 50(12), 2101-2113.

Boeckman, C. J. \& Bidwell, J. R. (2007). Spatial and Seasonal Variability in the Water Quality Characteristics of an Ephemeral Wetland. Proceedings of the Oklahoma Academy of Sciences 87, 45-54.

Buentello, J. A., Gatlin III, D. M., \& Neill, W. H. (2000). Effects of water temperature and dissolved oxygen on daily feed consumption, feed utilization and growth of channel catfish (Ictalurus punctatus). Aquaculture 182, 339-352.

Caballero, M., Lozano, S., Ortega, B., Urrutia, J., \& Macias, J. L. (1999). Environmental characteristics of Lake Tecocomulco, northern basin of Mexico, for the last 50000 years. Journal of Paleolimnology 22, 399-411.

Closs, G., Downes, B., \& Boulton, A. (2004). Freshwater ecology. Carlton, VIC: Blackwell.

Colwell, R. K. (2009). EstimateS: Statistical estimation of especies richness and shared species from samples. Versión 8. (Recuperado de <purl.oclc.org/ estimates $>$ ).

De la Lanza, E. G., \& Hernández-Pulido, S. (2005). Hidrogeología. In R. Huizar-Alvarez, E. J. JiménezFernández \& C. Juárez-López (Eds.), La Laguna de Tecocomulco geo-ecología de un desastre (pp. 109128). Ciudad de México, MEX: UNAM

Fontanarrosa, M. S., Chaparro, G. N. \& O'Farrell, I. (2012). Temporal and spatial patterns of macroinvertebrates associated with small and medium-sized free-floating plants. Wetlands, 33, 47-63.

García, E. (2004). Modificaciones al sistema de clasificación climática de Köppen. Ciudad de México: UNAM.
García-Palomo, P. A., \& Macías, J. L. (2005). Geologia y modelo geológico. In R. Huizar-Alvarez, E. J. Jiménez-Fernández \& C. Juárez-López (Eds.), La Laguna de Tecocomulco geo-ecología de un desastre (pp. 21-32). Ciudad de México: UNAM.

Guildford, S. J., \& Hecky, R. E. (2000). Total nitrogen, total phosphorus, and nutrient limitation in lakes and oceans: is there a common relationship? Journal of Limnology and Oceanography, 45(6), 1213-1223.

Gong, Z. J., Xie, P., \& Wang, S. D. (2000). Macrozoobentos in 2 shallow, mesotrophic Chinese lakes with contrasting sources of primary production. Journal of the North American Benthological Society, 19(4), 709-724.

Jeglum, J., Sandring, S., Christensen, P., Glimskär, A., Allard, A., Nilsson, L., \& Svensson, J. (2011). Main ecosystem characteristics and distribution of wetlands in Boreal and Alpine landscapes in Nothern Sweden under climate change. In O. Grillo \& G. Venora (Eds.), Ecosystems biodiversity (pp. 193218). Gornji Grad, GG: Intech.

Kandlec, R. H., \& Knight, R. L. (1996). Treatment wetlands. Boca Ratón, FLA: Lewis

Keeley, J. E., \& Zedler, P. H. (1998). Characterization and global distribution of vernal pools. Sacramento, CA: Native Plant Society.

Krantz, W. G., \& Evans, W. D. (2009). A manual of Acarology. Texas, TX: Texas Tech University Press.

Krebs, C. J. (1989). Ecological methodology. Nueva York, NY: Harper.

Latha, C., \& Thanga, V. S. (2010). Macroinvertebrate diversity of Veli and Kadinamkulam lakes, South Kerala, India. Journal of. Environmental. Biology, 31(4), 543-547.

Lock, K., Asenova, M., \& Goethals, P. L. M. (2011). Benthic macroinvertebrates as indicators of the water quality in Bulgaria: A case-study in the Iskar river basin. Limnologica 41(4), 334-338.

Lot-Helgueras, A., \& Novelo-Retana, A. (1978). Guías botánicas de excursiones en México: Laguna de Tecocomulco. Ciudad de México: Sociedad Botánica.

Lucca, J. V., Pamplin, P. A. Z., Gessner, Trivinho-Strixino, A. F. S., Spadano-Albuquerque, A. L, \& Rocha, O. (2010). Benthic macroinvertebrates of a tropical lake: Lake Caçó, MA, Brasil. Brazilian Journal of Biology, 70(3), 593-600.

Merrit, R. W., Cummins, K. W., \& Berg, M. B. (2008). An introduction to the Aquatic Insects of North America. Iowa, IA: Kendall

Mitsch, W. J., \& Gosselink, J. G. (2007). Wetlands. Hoboken, NJ: John Wiley and Sons.

Pamplin, P. A. Z., Almeida, T. M. C., \& Rocha, O. (2006). Composition and distribution of benthic 
macroinvertebrates in Americana Reservoir (SP, Brazil). Acta Limnologica Brasiliensia, 18(2), 121-132.

Pérez-Arteaga, A., Jackson, S. F., Carrera, E., \& Gaston, K. J. (2005). Priority sites for wildfowl conservation in Mexico. Animal Conservation, 8, 41-50.

Rosenberg, D. M., \& Resh, V. H. (1993). Introduction to Freshwater Biomonitoring and Benthic Macroinvertebrates. Nueva York, NY: Chapman.

Savage, A. A. (1990). The distribution of Corixidae in lakes and the ecological status of the North West Midlands meres. Field. Studies, 7, 516-530.

Sharma, R. C. \& Rawat, J. S. (2009). Monitoring of aquatic macroinvertebrates as bioindicator for assessing the health of wetlands: A case study in the central Himalayas, India. Ecological Indicators, 9, 118-28.

Stein, H., Springer, M., \& Kohlmann, B. (2008). Comparison of two sampling methods for biomonitoring using aquatic macroinvertebrates in the Dos Novillos River, Costa Rica. Journal of Ecological Engineering, 34(4), 267-275.

Thomaz, S. M., \& Cunha, E. R. (2010). The role of macrophytes in habitat structuring in aquatic ecosystems: Methods of measurement, causes and consequences on animal assemblages, composition and biodiversity. Acta Limnologica Brasiliensia, 22(2), 218-236.

Thorp, H. J., \& Covich, A. P. (2001). Ecology and Classification of North American Freshwater invertebrates. San Diego, CA: Academic Press.

Trigal-Domínguez, C., Frenández-Aláez, C., \& GarcíaCriado, F. (2009). Ecological assessment of highly heterogeneous systems: The importance of taxonómic sufficiency. Limnologica 4, 208-214.

Van den Berg, M. S., Coops, H., Meijer, M. L., Scheffer, M., \& Simons, J. (1998). Clear water associated with a dense Chara vegetation in the shallow and turbid Lake Veluwemeer, the Netherlands. In E. Jeppesen, Ma. Søndergaard, Mo. Søndergaard, \& K. Kristoffersen (Eds.), Structuring Role of Submerged Macrophytes in Lakes (pp. 339-352). Nueva York, NY: Ecological Studies.

Vance, C. C., Smith, S. M., Malcolm, J. R., Huber, J., \& Bellocq, M. I. (2007). Differences Between Forest Type and Vertical Strata in the Diversity and Composition of Hymenpteran Families and Mymarid Genera in Northeastern Temperate Forests. Environment. Entomology, 36(5), 1073-1083. 